

\title{
Twistors and supertwistors for exceptional field theory
}

\author{
Martin Cederwall \\ Dept. of Fundamental Physics, Chalmers University of Technology, \\ Gothenburg, SE 412 96 Sweden \\ E-mail: martin.cederwall@chalmers.se
}

ABSTRACT: As a means of examining the section condition and its possible solutions and relaxations, we perform twistor transforms related to versions of exceptional field theory with Minkowski signature. The spinor parametrisation of the momenta naturally solves simultaneously both the mass-shell condition and the (weak) section condition. It is shown that the incidence relations for multi-particle twistors force them to share a common section, but not to be orthogonal. The supersymmetric extension contains additional scalar fermionic variables shown to be kappa-symmetry invariants. We speculate on some implications, among them a possible relation to higher spin theory.

KeYwords: Superspaces, Space-Time Symmetries, M-Theory

ArXIV EPRINT: 1510.02298 


\section{Contents}

1 The section condition - background and motivation 1

2 The twistor variables $\quad 2$

$2.1 \mathrm{SL}(5) \quad 2$

$2.2 \operatorname{Spin}(5,5) \quad 4$

$2.3 E_{6(6)}$

$\begin{array}{lll}3 & \text { Supertwistors } & 6\end{array}$

$\begin{array}{lll}4 & \text { Outlook } & 7\end{array}$

\section{The section condition - background and motivation}

The section condition in doubled geometry [1-26] and exceptional geometry [27-46] is the subject of much discussion. On the one hand, it is indispensable for the gauge transformations - the generalised diffeomorphisms - to work, and thus it is integral to a geometric understanding of extended theories. On the other hand, this also means that not much is known of the geometric principles behind M-theory when one goes beyond the BPS sector where it is satisfied (massless modes on top of windings, roughly speaking). The stringtheoretic origin of the double field theory section condition is well understood, as it is a truncation of the level matching condition to this sector. No corresponding explanation of the section condition in exceptional field theory has been proposed.

The section condition is of course what locally makes the extended theory equivalent to a supergravity theory. Seen as a condition on generalised momenta (momenta and winding charges), it has a rôle as a BPS condition. The momenta are constrained to belong to non-maximal orbits under the "structure group" $\left(O(d, d)\right.$ or $\left.E_{n(n)}\right)$. Applied to a single momentum this is known as the "weak section condition", which for a momentum in the module $R_{1}$ reads

$$
\left.P^{2}\right|_{R_{2}}=0 \text {. }
$$

Here, $R_{1}$ and $R_{2}$ are the first two modules in the tensor hierarchy [47]. In a second-quantised theory, such a constraint does not make sense, since it does not respect multiplication of fields, and one is led to the "strong section condition", stating that any two derivatives, acting on any field (or gauge parameter) fulfill the relation

$$
\left.\partial \otimes \partial\right|_{R_{2}}=0
$$

This implies that all derivatives belong to a common "section", a maximal vector space of solutions. In a perturbative quantum theory, it is important not to over-interpret the 
constraint. A choice of global section is not allowed, and only stated sharing a common vertex in an amplitude diagram will obey a relative section condition [48]. Non-vanishing amplitudes, and terms in effective actions, may contain external momenta with no common section. Such a situation seems to go beyond proposals for mild relaxations of the section condition like the one by Lee [49]. It has indeed been appreciated that higher derivative terms typically call for modifications of the section condition [50].

\section{The twistor variables}

The idea of the present paper is to find a parametrisation of momenta in terms of twistor variables [51-61], which simultaneously solves the section condition and the mass-shell condition. This turns out to be natural in such a formalism (in fact, we are not aware of a reasonable way of similarly parametrising solutions to the section condition only). Indeed, it is only taken together that they carry meaning as a BPS condition. One may then consider going off-shell in twistor space, which typically entails going off the "spin shell". It is possible that systematic relaxation of the twistor constraints will lead to a kind of higher spin theory. In any case it looks like an interesting way of investigating the section condition and its possible relaxations.

We will also investigate how locality is implemented in twistor space through incidence relations, and show that they force multi-particle twistors to share a common section. They are however not forced to be orthogonal, which we take as a consistency check of the formalism. We will give the full details for the model case of $E_{4(4)} \approx \mathrm{SL}(5)$, and indicate more briefly how the transformations work for $E_{5(5)} \approx \operatorname{Spin}(5,5)$ and $E_{6(6)}$.

\section{$2.1 \quad \mathrm{SL}(5)$}

We now restrict to the structure group (corresponding to the duality group) $E_{4(4)} \approx \mathrm{SL}(5)$. Instead of letting the 4 dimensions of a vector space solution (the M-theory solution) to the section condition have Euclidean signature, as they have when they are internal coordinates in a compactification, we want Minkowski signature on solutions to the section condition. This can be achieved by choosing the local ("Lorentz") subgroup to be $\mathrm{SO}(2,3),{ }^{1}$ or, when spinors are included, its double cover $\operatorname{Spin}(2,3) \approx \operatorname{Sp}(4, \mathbf{R})$. Also in the type IIB solution [62], this real form allows for a section with Minkowski signature.

The momenta are a priori in the module 10 of SL(5), which can be written $P_{[m n]}$, or equivalently $P_{[a b]}$ or $P_{(\alpha \beta)}$, where $m, n=1, \ldots, 5$ are fundamental indices of $\mathrm{SL}(5)$, and $a, b=1, \ldots, 5$ and $\alpha, \beta=1, \ldots, 4$ vector and spinor indices, respectively, of $\operatorname{Spin}(2,3)$. It is assumed that there is some generalised vielbein (typically flat) to convert between curved and flat indices.

\footnotetext{
${ }^{1}$ This choice is not unique. Solutions to the weak section condition are elements in the Grassmannian of 2-planes in 5 dimensions. Vector spaces of solutions, i.e., solutions to the strong section condition, are planes intersection along a common line. If this line is time-like, Minkowski signature is obtained. The same signature may be obtained from $\mathrm{SO}(1,4)$. We prefer the present signuture, which allows for real spinors.
} 
The section condition and masslessness condition are

$$
\begin{aligned}
P_{[m n} P_{p q]} & =0, \\
P_{a b} P^{a b} & =0 .
\end{aligned}
$$

Note that the section condition is SL(5)-covariant, while the on-shell condition requires a generalised metric. In the following, I will treat them together and use fundamental $\mathrm{Sp}(4)$ indices. The two conditions are written collectively as

$$
\varepsilon^{\gamma \delta} P_{\alpha \gamma} P_{\beta \delta}=0
$$

where the section condition in $\mathbf{5}$ constitutes the $\varepsilon$-traceless part and $P^{2}=0$ the $\varepsilon$-trace. In this one-particle picture, it should be remembered that solutions to the section condition does not project down to a 4-dimensional subspace. Rather, we are dealing with the weak section condition, whose solutions form a real cône over the Grassmannian of 2-planes in 5 dimensions. This is a 7-dimensional space, and $P^{2}=0$ brings the dimension down to 6 . The dimension of the space of solutions always equals the dimension of $R_{1}$ under $E_{n-1}$.

We want a twistor parametrisation that solves the constraint (2.2) by expressing $P$ as a bilinear in a bosonic spinor. The dimensionality of the space of solutions tells us immediately that a single real $\Lambda_{\alpha}$ is not enough. A pair is the minimum, and we can put them in a complex spinor $\Lambda_{\alpha}$. The twistor parametrisation of the momentum is

$$
P_{\alpha \beta}=\Lambda_{(\alpha} \bar{\Lambda}_{\beta)}
$$

We now insert this into the constraint on $P$, eq. (2.2), and obtain

$$
\varepsilon^{\gamma \delta} P_{\alpha \gamma} P_{\beta \delta}=\varepsilon^{\gamma \delta} \Lambda_{(\alpha} \bar{\Lambda}_{\gamma)} \Lambda_{(\beta} \bar{\Lambda}_{\delta)}=-\frac{1}{2} \varepsilon^{\gamma \delta} \Lambda_{[\alpha} \bar{\Lambda}_{\beta]} \Lambda_{\gamma} \bar{\Lambda}_{\delta}
$$

In order for the constraints to be satisfied we need a constraint on $\Lambda$,

$$
\varepsilon^{\alpha \beta} \Lambda_{\alpha} \bar{\Lambda}_{\beta}=0
$$

Considering that the parametrisation of the momentum (2.3) also has a U(1) invariance under $\Lambda \rightarrow e^{i \theta} \Lambda$, the 6 degrees of freedom match the ones in $P$ counted earlier. Note also that the constraint (2.5) on $\Lambda$ is equally necessary in order to achieve the section condition and the on-shell constraint, so it seems that they are naturally linked together in a twistor description. Eq. (2.5) looks formally identical to the spin-shell constraint obtained from a massless twistor transform on $\mathrm{AdS}_{4}$ [61], which can be relaxed in order to obtain variables for higher spin theory. There, however, the spinors $\Lambda$ and $\bar{\Lambda}$ are conjugate to each other, and the constraint generates the $\mathrm{U}(1)$ transformation. Here, $\Lambda, \bar{\Lambda}$ describes only momenta, and gives a configuration space, not a phase space, for the twistors.

Introducing conjugate variables $W^{\alpha}$ to $\Lambda_{\alpha}$, the twistor transform is completed by

$$
W^{\alpha}=X^{\alpha \beta} \bar{\Lambda}_{\beta}
$$

From this, we derive the constraint

$$
\Lambda_{\alpha} W^{\alpha}-\bar{\Lambda}_{\alpha} \bar{W}^{\alpha}=0
$$


which is the generator of the $\mathrm{U}(1)$ transformation. It is also clear that the twistor transform is invariant under $X \rightarrow X+k P$, so that the choice of base-point $X$ for the world-line is irrelevant.

In twistor space, locality is represented in terms of incidence relations, some relations that tell us that $(\Lambda, W)$ and $\left(\Lambda^{\prime}, W^{\prime}\right)$ correspond to intersecting world-lines, i.e., that the respective transforms can be written using the same $X$. We find immediately that

$$
\Lambda_{\alpha} W^{\prime \alpha}-\bar{\Lambda}_{\alpha}^{\prime} \bar{W}^{\alpha}=0
$$

This is not the full answer, though. There will be new constraint in the two-particle phase space, obtained by acting with the generators (2.8) on the constraint (2.5). This gives a necessary completion of the incidence relations, namely

$$
\varepsilon^{\alpha \beta} \Lambda_{\alpha} \bar{\Lambda}_{\beta}^{\prime}=0
$$

We should now check that the strong version of the section condition is satisfied, i.e., that

$$
\varepsilon^{\gamma \delta} P_{[\alpha|\gamma|} P_{\beta] \delta}^{\prime}-\frac{1}{4} \varepsilon_{\alpha \beta} \varepsilon^{\gamma \delta} \varepsilon^{\varepsilon \varphi} P_{\gamma \varepsilon} P_{\delta \varphi}^{\prime}=0
$$

Here, it is important that the $\varepsilon$-trace remains non-vanishing — we want generically to have $P \cdot P^{\prime} \neq 0$ for the two momenta, only that they lie in the same linear subspace corresponding to a solution to the strong section condition, i.e., $P_{[m n} P_{p q]}^{\prime}=0$. Using the constraint (2.9) together with the constraints (2.5) on $\Lambda$ and $\Lambda^{\prime}$, we obtain

$$
\varepsilon^{\gamma \delta} P_{[\alpha|\gamma|} P_{\beta] \delta}^{\prime} \sim \varepsilon^{\gamma \delta} \Lambda_{[\alpha} \Lambda_{\beta}^{\prime} \bar{\Lambda}_{\gamma} \bar{\Lambda}_{\delta]}^{\prime} \sim \varepsilon_{\alpha \beta} \varepsilon^{\gamma \delta} \Lambda_{\gamma} \Lambda_{\delta}^{\prime} \varepsilon^{\varepsilon \varphi} \bar{\Lambda}_{\varepsilon} \bar{\Lambda}_{\varphi}^{\prime}
$$

Antisymmetrisation in four indices implies that the expression is pure $\varepsilon$-trace. (Note that the expression vanishes if the primes are removed.)

We find it very encouraging, indeed a decisive test of the relevance of the formalism for exceptional geometry, that the simplest possible form of incidence relations, reducing to the constraints on a single twistor for coinciding spinors, does precisely what is wanted, namely solving the strong section condition without yielding orthogonal momenta.

\section{$2.2 \operatorname{Spin}(5,5)$}

The twistor transform for the case of structure group $\operatorname{Spin}(5,5)$ will now be described. In order to have a section with Minkowski signature, the local subgroup is chosen to be $\mathrm{USp}(2,2) \times \mathrm{USp}(2,2)$. Each factor has an invariant antisymmetric tensor $\epsilon_{a b}$ and a metric $\eta_{a \bar{b}}$ with signature $(2,2)$. Then the Lorentz group of the section is the diagonal subgroup $\operatorname{USp}(2,2) \approx \operatorname{Spin}(1,4)$.

The momentum, a chiral spinor 16 under $\operatorname{Spin}(5,5)$, is in the bi-fundamental $(\mathbf{4}, \mathbf{4})$ under $\operatorname{USp}(2,2) \times \operatorname{USp}(2,2)$. Even though the fundamental is complex, the bi-fundamental is pseudo-real, thanks to the existence of the involution

$$
v_{a a^{\prime}} \rightarrow \tilde{v}_{a a^{\prime}}=(\sigma(v))_{a a^{\prime}}=\eta_{a \bar{a}} \eta_{a^{\prime} \bar{a}^{\prime}} \epsilon^{\bar{a} \bar{b}} \epsilon^{\bar{a}^{\prime} \bar{b}^{\prime}} \bar{v}_{\bar{b} \bar{b}^{\prime}} .
$$


We can choose $\tilde{P}=P$. The weak section condition is in $\mathbf{1 0}$ of $\operatorname{Spin}(5,5)$, and states that $P$ is a pure spinor. Together with the condition $P^{2}=0$, the constraints read

$$
\epsilon^{a^{\prime} b^{\prime}} P_{a a^{\prime}} P_{b b^{\prime}}=0, \quad \epsilon^{a b} P_{a a^{\prime}} P_{b b^{\prime}}=0 .
$$

The twistor solution of these constraints requires an object in the fundamental of each component, i.e., $\lambda_{a}$ and $\mu_{a^{\prime}}$, with the momentum formed as

$$
P=\lambda \mu^{t}+\sigma\left(\lambda \mu^{t}\right) .
$$

This gives 16 real degrees of freedom. The momentum is invariant under the $\mathrm{SU}(2) \times \mathbf{R}^{+}$ transformations

$$
\begin{aligned}
& (\lambda, \tilde{\lambda}) \rightarrow(\lambda, \tilde{\lambda}) M \\
& (\mu, \tilde{\mu}) \rightarrow(\mu, \tilde{\mu})\left(M^{-1}\right)^{t}
\end{aligned}
$$

where the matrix $M$ is give by

$$
M=\left[\begin{array}{cc}
\alpha & -\bar{\beta} \\
\beta & \bar{\alpha}
\end{array}\right],
$$

and where $\tilde{\lambda}_{a}=\eta_{a \bar{a}} \epsilon^{\bar{a} \bar{b}} \bar{\lambda}_{\bar{b}}$ and $\alpha, \beta \in \mathbf{C}$. In order for the transform (2.14) to solve the constraints $(2.13)$, the twistor variables need to satisfy the scalar constraints

$$
\eta^{a \bar{a}} \lambda_{a} \bar{\lambda}_{\bar{a}}=0, \quad \eta^{a^{\prime} \bar{a}^{\prime}} \mu_{a^{\prime}} \bar{\mu}_{\bar{a}^{\prime}}=0
$$

The number of on-shell twistor degrees of freedom is $16-4-2=10$, matching those of the null pure spinor $P$. The discussion of incidence relations etc. can be performed in analogy with the $n=4$ case, and the details will not be given here.

\section{$2.3 \quad E_{6(6)}$}

For $n=6$, the structure group is $E_{6(6)}$. The locally realised group leading to a section with Minkowski signature is $\operatorname{USp}(4,4)$ (with maximal compact subgroup $\operatorname{Spin}(5) \times \operatorname{Spin}(5)$ ). It is convenient to realise this group as an orthogonal group over the quaternions, $\operatorname{USp}(4,4) \approx$ $\operatorname{Spin}(2,2 ; \mathbf{H})$. Then, as usual $[55,63,64]$, the $\mathrm{SU}(2) \mathrm{R}$-symmetry is realised by right multiplication with unit quaternions. This is a convenient way of manifesting the (pseudo-)reality of the fundamental $(\mathbf{8}, \mathbf{2})$, equivalent to an "SU(2) Majorana condition".

A momentum in $\mathbf{2 7}$ of $E_{6(6)}$ becomes a hermitean and traceless $(4 \times 4)$-matrix with entries in $\mathbf{H}$. The constraints on $P$ (the section condition together with " $P^{2}=0$ ") then simply read

$$
P^{2}=0
$$

where quaternionic matrix multiplication is implied. The solution space is 16-dimensional, and consists of null elements in a cone over the Cayley plane [36, 65].

A single "spinor" in $(\mathbf{8}, \mathbf{2})$ of $\mathrm{USp}(4,4) \times \mathrm{SU}(2)$ is not enough, at least two are needed. The R-symmetry then becomes $\operatorname{Spin}(2, \mathbf{H}) \approx \mathrm{USp}(4) \approx \operatorname{Spin}(5)$. We represent this "spinor" $\Lambda$ as a $(4 \times 2)$-matrix. This means that a parametrisation

$$
P=\Lambda \Lambda^{\dagger}
$$


will have an invariance under $\Lambda \rightarrow \Lambda M$, where $M$ is a matrix in $\operatorname{Spin}(2 ; \mathbf{H})$, i.e., $M M^{\dagger}=$ $\mathbb{1}$ [63]. This takes away 10 degrees of freedom. In order for the constraint (2.18) on $P$ to be satisfied, $\Lambda$ has to obey the 6 constraints (in a hermitean $(2 \times 2)$-matrix)

$$
\Lambda^{\dagger} \Lambda=0
$$

Strictly speaking, the trace should have been subtracted in eq. (2.19), but it already vanishes due to eq. (2.20). The counting of the twistor degrees of freedom now gives $32-10-6=16$, matching the ones in $P$.

\section{Supertwistors}

It is quite straightforward to extend the construction to supersymmetric particles. The fermionic variables in the supertwistor will arise as invariants under $\kappa$-symmetry. It is therefore desirable to start from an action to be able to keep proper track of the local symmetries, especially $\kappa$-symmetry. The alternative would be to perform the supersymmetric extension more ad hoc in the twistor formalism, which seems less satisfactory. This can of course also be done for bosonic particles.

The construction will be performed specifically for the SL(5) case, and for minimal supersymmetry. The superparticle action should depend only on the combination

$$
\Pi^{\alpha \beta}=\dot{X}^{\alpha \beta}+\theta^{(\alpha} \dot{\theta}^{\beta)} .
$$

It will eventually equal the momentum. It is invariant under the global supersymmetry transformations

$$
\begin{aligned}
\delta_{\epsilon} X^{\alpha \beta} & =-\epsilon^{(\alpha} \theta^{\beta)}, \\
\delta_{\epsilon} \theta^{\alpha} & =\epsilon^{\alpha} .
\end{aligned}
$$

The weak section condition and the masslessness condition must follow from the action, and are implemented by the introduction of a set of Lagrange multipliers $V_{\alpha \beta}$ in an antisymmetric matrix. The action is

$$
S=\frac{1}{2} \int d \tau V_{\alpha \beta} V_{\gamma \delta} \Pi^{\alpha \gamma} \Pi^{\beta \delta} .
$$

The $V$ 's are non-dynamical, and as long as they are assumed to be non-degenerate, can be gauge fixed to $\epsilon$, using the symmetry generated by the primary constraint $P_{V}^{\alpha \beta} \approx 0$. All following equations are given after that gauge fixing. Clearly, the momentum conjugate to $X^{\alpha \beta}$ is $P_{\alpha \beta}=\epsilon_{\alpha \gamma} \epsilon_{\beta \delta} \Pi^{\gamma \delta}$, and the constraints (2.2) are reproduced - they are the equations of motion obtained by variation of the Lagrange multipliers. In addition, the momentum $\pi_{\alpha}$ conjugate to $\theta^{\alpha}$ is constrained by

$$
\pi_{\alpha}-P_{\alpha \beta} \theta^{\beta} \approx 0
$$

It is obvious that the momentum, obeying (2.2), will have vanishing determinant, so some of the fermionic constraints are first class, generating $\kappa$-symmetry. It is easily checked that $P$ has half rank precisely when eq. (2.2) is satisfied, leading to half-BPS excitations, and 
reducing the dynamics to that of an ordinary superparticle in 4 dimensions. The (local) $\kappa$-symmetry may also be verified directly in the action, by inserting

$$
\begin{aligned}
\delta_{\kappa} X^{\alpha \beta} & =\kappa^{(\alpha} \theta^{\beta)}, \\
\delta_{\kappa} \theta^{\alpha} & =\kappa^{\alpha},
\end{aligned}
$$

where $P_{\alpha \beta} \kappa^{\beta}=0$. Solving this condition with $\kappa^{\alpha}=\Pi^{\alpha \beta} \varrho_{\beta}$ and inserting the variations in the action gives a result that vanishes modulo constraints.

The twistor parametrisation of the bosonic momentum is identical to the bosonic twistor transform, eq. (2.3). The relation between the conjugate twistor variables $W$ and the original superspace variables has to be modified, however. It reads

$$
W^{\alpha}=X^{\alpha \beta} \bar{\Lambda}_{\beta}+\theta^{\alpha} \bar{\xi},
$$

where the fermionic variables are constructed as

$$
\xi=\frac{1}{2} \Lambda_{\alpha} \theta^{\alpha}, \quad \bar{\xi}=\frac{1}{2} \bar{\Lambda}_{\alpha} \theta^{\alpha} .
$$

The fermionic variables are easily shown to be invariant under $\kappa$-symmetry, precisely thanks to the constraint on $\Lambda$, eq. (2.5). They are conjugate to each other, $\{\xi, \bar{\xi}\}=1$ and span the full fermionic phase space. Global supersymmetry transforms the supertwistor variables according to

$$
\begin{aligned}
\delta_{\epsilon} \Lambda_{\alpha} & =0, \\
\delta_{\epsilon} W^{\alpha} & =\frac{1}{2} \epsilon^{\alpha} \bar{\xi}, \\
\delta_{\epsilon} \xi & =\frac{1}{2} \epsilon^{\alpha} \Lambda_{\alpha} .
\end{aligned}
$$

\section{Outlook}

We have constructed twistor transforms for exceptional field theory with structure group $E_{n(n)}, n=4,5,6$. The main idea is that the section condition and the on-shell condition are natural to treat together.

It is unclear if the series can be continued to higher $n$ (lower $n$ should be simple), but we have so far not been able to perform the construction for $n=7$. This may be connected to the observation that, in the range where the construction has been worked out, the number of real components in an unconstrained twistor $\Lambda$ is $2^{n-1}$. Already at $n=6$, this number is 32 and the R-symmetry is Spin(5), which can be identified with the rotation group of 5 extra coordinates. For $n=7$, the size of the module needed seems to go beyond the M-theory spinor at maximal supersymmetry. The corresponding procedure in double field theory is the somewhat trivial procedure of performing separate twistor transforms in the two sectors of $O(1, d-1) \times O(1, d-1) \subset O(d, d)$.

Another limitation is that we have only considered "internal" directions, although in Minkowski signature, and left the remaining $11-n$ directions out of the picture. Including them would modify the on-shell condition in a way that will also change the twistor transform. 
Supersymmetry and superfields in flat superspace is straightforward for the $E_{n(n)}$ structure groups. Supermultiplets have been constructed in component language in a number of papers, e.g. refs. [35, 39, 43]. Giving a geometric meaning to exceptional superspace seems more difficult, although some progress has been made in double supergeometry [66]. A very desirable goal would be an understanding of the structure corresponding to pure spinors for ordinary superspace and supergeometry [67-75]. It seems that precisely the section condition stands in the way, and needs to be better understood for this goal to be attained. If at some point the issue is resolved, it should be possible to construct off-shell supersymmetric actions for extended supersymmetric field theory and supergeometry along the lines of refs. [76-80].

We do not expect the results to have direct bearing on calculations or on construction of extended field theories. Rather they may provide an interesting message for field theory and geometry: that the section condition ultimately should be taken seriously and arise as equations of motion, on equal footing with " $P^{2}=0$ ". We do not claim that the naïve way the weak section condition is obtained in section 3 - from Lagrange multipliers in a world-line formalism - has any direct connection to such a field theory formalism; it is practical rather than deep. The results may however give some direction concerning possible relaxation of the section condition, in its weak or strong version. Going off-shell in the twistor formalism means including an infinite number of fields with different spin. For ordinary higher spin theory [81-83], this is a natural way to derive a set of variables (oscillators) for the field theory. Although this applies to AdS twistors [59-61], a similar statement could be valid in M-theory, and the present formalism seems to provide a possible starting point for an investigation of this issue.

\section{Acknowledgments}

The author would like to thank the Mainz Institute for Theoretical Physics (MITP) for its hospitality and support during the programme "Stringy geometry", where part of this work was done.

Open Access. This article is distributed under the terms of the Creative Commons Attribution License (CC-BY 4.0), which permits any use, distribution and reproduction in any medium, provided the original author(s) and source are credited.

\section{References}

[1] M.J. Duff, Duality Rotations in String Theory, Nucl. Phys. B 335 (1990) 610 [InSPIRE].

[2] A.A. Tseytlin, Duality symmetric closed string theory and interacting chiral scalars, Nucl. Phys. B 350 (1991) 395 [inSPIRE].

[3] W. Siegel, Two-vierbein formalism for string-inspired axionic gravity, Phys. Rev. D 47 (1993) 5453 [hep-th/9302036] [INSPIRE].

[4] W. Siegel, Superspace duality in low-energy superstrings, Phys. Rev. D 48 (1993) 2826 [hep-th/9305073] [INSPIRE]. 
[5] W. Siegel, Manifest duality in low-energy superstrings, in the proceedings of the International Conference on Strings 93, Berkeley, California, U.S.A., May 24-29 1993 [hep-th/9308133] [INSPIRE].

[6] C.M. Hull, A Geometry for non-geometric string backgrounds, JHEP 10 (2005) 065 [hep-th/0406102] [INSPIRE].

[7] C.M. Hull, Doubled Geometry and T-Folds, JHEP 07 (2007) 080 [hep-th/0605149] [INSPIRE].

[8] C. Hull and B. Zwiebach, Double Field Theory, JHEP 09 (2009) 099 [arXiv:0904.4664] [INSPIRE].

[9] O. Hohm, C. Hull and B. Zwiebach, Background independent action for double field theory, JHEP 07 (2010) 016 [arXiv: 1003.5027] [INSPIRE].

[10] O. Hohm, C. Hull and B. Zwiebach, Generalized metric formulation of double field theory, JHEP 08 (2010) 008 [arXiv: 1006.4823] [INSPIRE].

[11] A. Coimbra, C. Strickland-Constable and D. Waldram, Supergravity as Generalised Geometry I: Type II Theories, JHEP 11 (2011) 091 [arXiv:1107.1733] [INSPIRE].

[12] O. Hohm and S.K. Kwak, Frame-like Geometry of Double Field Theory, J. Phys. A 44 (2011) 085404 [arXiv: 1011.4101] [INSPIRE].

[13] O. Hohm and S.K. Kwak, $N=1$ Supersymmetric Double Field Theory, JHEP 03 (2012) 080 [arXiv:1111.7293] [INSPIRE].

[14] I. Jeon, K. Lee and J.-H. Park, Differential geometry with a projection: Application to double field theory, JHEP 04 (2011) 014 [arXiv: 1011.1324] [INSPIRE].

[15] I. Jeon, K. Lee and J.-H. Park, Stringy differential geometry, beyond Riemann, Phys. Rev. D 84 (2011) 044022 [arXiv:1105.6294] [InSPIRE].

[16] I. Jeon, K. Lee and J.-H. Park, Supersymmetric Double Field Theory: Stringy Reformulation of Supergravity, Phys. Rev. D 85 (2012) 081501 [Erratum ibid. D 86 (2012) 089903] [arXiv: 1112.0069] [INSPIRE].

[17] O. Hohm and B. Zwiebach, Large Gauge Transformations in Double Field Theory, JHEP 02 (2013) 075 [arXiv:1207.4198] [INSPIRE].

[18] J.-H. Park, Comments on double field theory and diffeomorphisms, JHEP 06 (2013) 098 [arXiv: 1304.5946] [INSPIRE].

[19] D.S. Berman, M. Cederwall and M.J. Perry, Global aspects of double geometry, JHEP 09 (2014) 066 [arXiv: 1401.1311] [INSPIRE].

[20] M. Cederwall, The geometry behind double geometry, JHEP 09 (2014) 070 [arXiv: 1402.2513] [INSPIRE].

[21] O. Hohm, D. Lüst and B. Zwiebach, The Spacetime of Double Field Theory: Review, Remarks and Outlook, Fortsch. Phys. 61 (2013) 926 [arXiv:1309.2977] [INSPIRE].

[22] G. Papadopoulos, Seeking the balance: Patching double and exceptional field theories, JHEP 10 (2014) 089 [arXiv: 1402.2586] [INSPIRE].

[23] C.M. Hull, Finite Gauge Transformations and Geometry in Double Field Theory, JHEP 04 (2015) 109 [arXiv: 1406.7794] [inSPIRE].

[24] M. Cederwall, T-duality and non-geometric solutions from double geometry, Fortsch. Phys. 62 (2014) 942 [arXiv: 1409.4463] [INSPIRE]. 
[25] R. Blumenhagen, F. Hassler and D. Lüst, Double Field Theory on Group Manifolds, JHEP 02 (2015) 001 [arXiv: 1410.6374] [InSPIRE].

[26] R. Blumenhagen, P. du Bosque, F. Hassler and D. Lüst, Generalized Metric Formulation of Double Field Theory on Group Manifolds, JHEP 08 (2015) 056 [arXiv:1502.02428] [INSPIRE].

[27] C.M. Hull, Generalised Geometry for M-theory, JHEP 07 (2007) 079 [hep-th/0701203] [INSPIRE].

[28] P.P. Pacheco and D. Waldram, M-theory, exceptional generalised geometry and superpotentials, JHEP 09 (2008) 123 [arXiv:0804.1362] [INSPIRE].

[29] C. Hillmann, Generalized $E_{7(7)}$ coset dynamics and D = 11 supergravity, JHEP 03 (2009) 135 [arXiv:0901.1581] [INSPIRE].

[30] D.S. Berman and M.J. Perry, Generalized Geometry and M-theory, JHEP 06 (2011) 074 [arXiv: 1008.1763] [INSPIRE].

[31] D.S. Berman, H. Godazgar and M.J. Perry, $\mathrm{SO}(5,5)$ duality in M-theory and generalized geometry, Phys. Lett. B 700 (2011) 65 [arXiv:1103.5733] [InSPIRE].

[32] D.S. Berman, H. Godazgar, M. Godazgar and M.J. Perry, The Local symmetries of M-theory and their formulation in generalised geometry, JHEP 01 (2012) 012 [arXiv:1110.3930] [INSPIRE].

[33] D.S. Berman, H. Godazgar, M.J. Perry and P. West, Duality Invariant Actions and Generalised Geometry, JHEP 02 (2012) 108 [arXiv:1111.0459] [INSPIRE].

[34] A. Coimbra, C. Strickland-Constable and D. Waldram, $E_{d(d)} \times \mathbb{R}^{+}$generalised geometry, connections and M-theory, JHEP 02 (2014) 054 [arXiv:1112.3989] [INSPIRE].

[35] A. Coimbra, C. Strickland-Constable and D. Waldram, Supergravity as Generalised Geometry II: $E_{d(d)} \times \mathbb{R}^{+}$and M-theory, JHEP 03 (2014) 019 [arXiv:1212.1586] [InSPIRE].

[36] D.S. Berman, M. Cederwall, A. Kleinschmidt and D.C. Thompson, The gauge structure of generalised diffeomorphisms, JHEP 01 (2013) 064 [arXiv: 1208.5884] [INSPIRE].

[37] J.-H. Park and Y. Suh, U-geometry: SL(5), JHEP 04 (2013) 147 [Erratum ibid. 11 (2013) 210] [arXiv: 1302.1652] [INSPIRE].

[38] M. Cederwall, J. Edlund and A. Karlsson, Exceptional geometry and tensor fields, JHEP 07 (2013) 028 [arXiv: 1302.6736] [INSPIRE].

[39] M. Cederwall, Non-gravitational exceptional supermultiplets, JHEP 07 (2013) 025 [arXiv: 1302.6737] [INSPIRE].

[40] M. Cederwall, M-branes on U-folds, in the proceedings of the 7th International Workshop on Supersymmetries and Quantum Symmetries (SQS 07), Dubna, Russia, July 30-August 4 2007 [arXiv: 0712.4287] [INSPIRE].

[41] G. Aldazabal, M. Graña, D. Marqués and J.A. Rosabal, Extended geometry and gauged maximal supergravity, JHEP 06 (2013) 046 [arXiv: 1302.5419] [INSPIRE].

[42] G. Aldazabal, M. Graña, D. Marqués and J.A. Rosabal, The gauge structure of Exceptional Field Theories and the tensor hierarchy, JHEP 04 (2014) 049 [arXiv: 1312.4549] [INSPIRE].

[43] O. Hohm and H. Samtleben, Exceptional Field Theory I: $E_{6(6)}$ covariant Form of M-theory and Type IIB, Phys. Rev. D 89 (2014) 066016 [arXiv:1312.0614] [INSPIRE].

[44] O. Hohm and H. Samtleben, Exceptional field theory. II. E $E_{7(7)}$, Phys. Rev. D 89 (2014) 066017 [arXiv: 1312 .4542] [INSPIRE]. 
[45] O. Hohm and H. Samtleben, Exceptional field theory. III. E $E_{8(8)}$, Phys. Rev. D 90 (2014) 066002 [arXiv: 1406.3348] [INSPIRE].

[46] M. Cederwall and J.A. Rosabal, E8 geometry, JHEP 07 (2015) 007 [arXiv: 1504.04843] [INSPIRE].

[47] B. de Wit, H. Nicolai and H. Samtleben, Gauged Supergravities, Tensor Hierarchies and M-theory, JHEP 02 (2008) 044 [arXiv:0801.1294] [INSPIRE].

[48] G. Bossard and A. Kleinschmidt, Loops in exceptional field theory, arXiv:1510.07859 [INSPIRE].

[49] K. Lee, Towards Weakly Constrained Double Field Theory, arXiv:1509.06973 [INSPIRE].

[50] O. Hohm, W. Siegel and B. Zwiebach, Doubled $\alpha^{\prime}$-geometry, JHEP 02 (2014) 065 [arXiv: 1306.2970] [INSPIRE].

[51] R. Penrose and M.A.H. MacCallum, Twistor theory: An Approach to the quantization of fields and space-time, Phys. Rept. 6 (1972) 241 [INSPIRE].

[52] A. Ferber, Supertwistors and Conformal Supersymmetry, Nucl. Phys. B 132 (1978) 55 [INSPIRE].

[53] T. Shirafuji, Lagrangian Mechanics of Massless Particles With Spin, Prog. Theor. Phys. 70 (1983) 18 [INSPIRE].

[54] A.K.H. Bengtsson, I. Bengtsson, M. Cederwall and N. Linden, Particles, Superparticles and Twistors, Phys. Rev. D 36 (1987) 1766 [InSPIRE].

[55] I. Bengtsson and M. Cederwall, Particles, Twistors and the Division Algebras, Nucl. Phys. B 302 (1988) 81 [INSPIRE].

[56] N. Berkovits, A Supertwistor Description of the Massless Superparticle in Ten-dimensional Superspace, Phys. Lett. B 247 (1990) 45 [InSPIRE].

[57] M. Cederwall, Octonionic particles and the $S_{7}$ symmetry, J. Math. Phys. 33 (1992) 388 [INSPIRE].

[58] M. Cederwall, Introduction to division algebras, sphere algebras and twistors, hep-th/9310115 [INSPIRE].

[59] P. Claus, M. Günaydın, R. Kallosh, J. Rahmfeld and Y. Zunger, Supertwistors as quarks of $\mathrm{SU}(2,2 \mid 4)$, JHEP 05 (1999) 019 [hep-th/9905112] [INSPIRE].

[60] M. Cederwall, Geometric construction of AdS twistors, Phys. Lett. B 483 (2000) 257 [hep-th/0002216] [INSPIRE].

[61] M. Cederwall, AdS twistors for higher spin theory, AIP Conf. Proc. 767 (2005) 96 [hep-th/0412222] [INSPIRE].

[62] C.D.A. Blair, E. Malek and J.-H. Park, M-theory and Type IIB from a Duality Manifest Action, JHEP 01 (2014) 172 [arXiv: 1311.5109] [INSPIRE].

[63] A. Sudbery, Division algebras, (pseudo)orthogonal groups and spinors, J. Phys. A 17 (1984) 939.

[64] T. Kugo and P.K. Townsend, Supersymmetry and the Division Algebras, Nucl. Phys. B 221 (1983) 357 [INSPIRE].

[65] M. Cederwall, Jordan algebra dynamics, Phys. Lett. B 210 (1988) 169 [InSPIRE].

[66] M. Hatsuda, K. Kamimura and W. Siegel, Superspace with manifest T-duality from type-II superstring, JHEP 06 (2014) 039 [arXiv:1403.3887] [INSPIRE]. 
[67] B.E.W. Nilsson, Pure Spinors as Auxiliary Fields in the Ten-dimensional Supersymmetric Yang-Mills Theory, Class. Quant. Grav. 3 (1986) L41 [inSPIRE].

[68] P.S. Howe, Pure spinors lines in superspace and ten-dimensional supersymmetric theories, Phys. Lett. B 258 (1991) 141 [inSPIRE].

[69] P.S. Howe, Pure spinors, function superspaces and supergravity theories in ten-dimensions and eleven-dimensions, Phys. Lett. B 273 (1991) 90 [INSPIRE].

[70] N. Berkovits, Super Poincaré covariant quantization of the superstring, JHEP 04 (2000) 018 [hep-th/0001035] [INSPIRE].

[71] N. Berkovits, Covariant quantization of the superparticle using pure spinors, JHEP 09 (2001) 016 [hep-th/0105050] [INSPIRE].

[72] M. Cederwall, B.E.W. Nilsson and D. Tsimpis, The Structure of maximally supersymmetric Yang-Mills theory: Constraining higher order corrections, JHEP 06 (2001) 034 [hep-th/0102009] [INSPIRE].

[73] M. Cederwall, B.E.W. Nilsson and D. Tsimpis, $D=10$ super Yang-Mills at $O\left(\alpha^{\prime 2}\right)$, JHEP 07 (2001) 042 [hep-th/0104236] [INSPIRE].

[74] M. Cederwall, Superspace methods in string theory, supergravity and gauge theory, in the proceedings of the 37th Karpacz Winter School of Theoretical Physics: New Developments in Fundamental Interactions Theories, Karpacz, Poland, February 6-15 2001 [hep-th/0105176] [INSPIRE].

[75] M. Cederwall, B.E.W. Nilsson and D. Tsimpis, Spinorial cohomology and maximally supersymmetric theories, JHEP 02 (2002) 009 [hep-th/0110069] [INSPIRE].

[76] M. Cederwall, Towards a manifestly supersymmetric action for 11-dimensional supergravity, JHEP 01 (2010) 117 [arXiv:0912.1814] [INSPIRE].

[77] M. Cederwall, D = 11 supergravity with manifest supersymmetry, Mod. Phys. Lett. A 25 (2010) 3201 [arXiv: 1001.0112] [INSPIRE].

[78] M. Cederwall, $N=8$ superfield formulation of the Bagger-Lambert-Gustavsson model, JHEP 09 (2008) 116 [arXiv:0808.3242] [inSPIRE].

[79] M. Cederwall, Superfield actions for $N=8$ and $N=6$ conformal theories in three dimensions, JHEP 10 (2008) 070 [arXiv: 0809.0318] [INSPIRE].

[80] M. Cederwall, Pure spinor superfields - an overview, Springer Proc. Phys. 153 (2014) 61 [arXiv: 1307.1762] [INSPIRE].

[81] I.A. Bandos, J. Lukierski and D.P. Sorokin, Superparticle models with tensorial central charges, Phys. Rev. D 61 (2000) 045002 [hep-th/9904109] [InSPIRE].

[82] M.A. Vasiliev, Conformal higher spin symmetries of $4 D$ massless supermultiplets and $\operatorname{osp}(L, 2 M)$ invariant equations in generalized (super)space, Phys. Rev. D 66 (2002) 066006 [hep-th/0106149] [INSPIRE].

[83] O.A. Gelfond and M.A. Vasiliev, $\mathrm{Sp}(8)$ invariant higher spin theory, twistors and geometric BRST formulation of unfolded field equations, JHEP 12 (2009) 021 [arXiv:0901.2176] [INSPIRE]. 